\title{
Introducer Sheath Compatibility
}

National Cancer Institute

\section{Source}

National Cancer Institute. Introducer Sheath Compatibility. NCI Thesaurus. Code C150144.

The size of the device that would fit through an introducer sheath. 